Journal of Environmental
Analysis and Progress

\title{
Internet das coisas na gestão de resíduos sólidos: revisão sistemática com análise bibliométrica da literatura
}

\section{Internet of things in solid waste management: systematic review with bibliometric analysis of the literature}

\author{
Wilson Ramos Aragão Júnior ${ }^{\mathrm{a}}$, Antônio Italcy de Oliveira Júnior ${ }^{\mathrm{a}}$ \\ ${ }^{a}$ Universidade Federal de Pernambuco-UFPE. Av. Prof. Moraes Rego, 1235, Cidade Universitária, Recife, Pernambuco, \\ Brasil. CEP: 50670-901. E-mail: wilsonramosaragao@ hotmail.com, antonioitalcy@ hotmail.com.
}

\begin{tabular}{l}
\hline A R T I C L E I N F O \\
\hline Recebido 01 Abr 2021 \\
Aceito 14 Jun 2021 \\
Publicado 06 Set 2021
\end{tabular}

\begin{abstract}
A B S T R A C T
The Internet of Things (IoT) is a technology of Industry 4.0 that is currently being pointed out as a mechanism capable of helping construct intelligent and sustainable cities. Within this context, solid waste management (SW) is one of the problems of cities to be solved. Thus, this article carried out a systematic review with bibliometric analysis to understand the relationship and IoT application in SW management. The work is organized in four stages: Searching articles in the database, Meta-analysis, Bibliometric, and Systematic Analysis. The data search was carried out in the Scopus database, selecting the articles using the PRISMA method. In the meta-analysis, bibliometric and publication-specific data were extracted. The bibliometric analysis stage was performed quantitatively and qualitatively using software VOSviewer e Microsoft Excel. In the systematic analysis, the information contained in the works was synthesized. All 28 articles included in the analysis were published between 2013 and 2020. The majority of authors and institutions come from countries in Asia and Europe, demonstrating the need for global expansion of studies. In the coauthorship and citation networks, there was little relationship between the authors of the documents. Regarding the co-occurrence networks between the words, there was a focus on the use of IoT technologies to automate dumps to improve the management of SW. It became evident the great relevance of IoT in the management of SW for the construction of intelligent and sustainable cities.
\end{abstract}

Keywords: Industry 4.0, IoT, smart cities, sustainable development, PRISMA method.

\section{R E S U M O}

A Internet das Coisas (IoT) é uma tecnologia da Indústria 4.0 que vem sendo apontada na atualidade como um mecanismo capaz de auxiliar na construção das cidades inteligentes e sustentáveis. Dentro deste contexto, a gestão de resíduos sólidos (RS) é um dos problemas das cidades a serem solucionados. Assim, este artigo realizou uma revisão sistemática com análise bibliométrica, buscando compreender a relação e a aplicação da IoT na gestão de RS. O trabalho foi organizado em quatro etapas: Busca de artigos em base de dados, Meta-análise, Análises Bibliométrica e Sistemática. A busca de dados foi realizada na base da Scopus, selecionando os artigos usando o método PRISMA. Na meta-análise foram extraídos os dados bibliométricos e específicos das publicações. A etapa de análise bibliométrica foi realizada quantitativamente e qualitativamente usando os softwares VOSviewer e Microsoft Excel. Na análise sistemática, a informação contida nos estudos foi sintetizada. Os 28 artigos incluídos na análise foram publicados entre 2013 e 2020. A maioria dos autores e das instituições é originária de países da Ásia e da Europa, demonstrando a necessidade de ampliação global de estudos. Nas redes de coautoria e de citação, observou-se pouca relação entre os autores dos documentos. Em relação as redes de coocorrência entre as palavras, notou-se enfoque 
na utilização de tecnologias de IoT para automatização de lixeiras com intuito de melhorar o gerenciamento dos RS. Ficou evidente a grande relevância do emprego da IoT na gestão de RS para construção de cidades inteligentes e sustentáveis.

Palavras-Chave: Indústria 4.0, IoT, cidades inteligentes, desenvolvimento sustentável, método PRISMA.

\section{Introdução}

O crescimento urbano, populacional e econômico acelerado é uma realidade na maioria das nações. $\mathrm{O}$ avanço das civilizações, na atualidade gera, consequentemente, grandes impactos negativos no meio ambiente e na saúde dos indivíduos. Este cenário torna-se ainda mais agravante em países emergentes como, por exemplo, o Brasil onde existe elevada desigualdade socioeconômica. As impactantes transformações oriundas do ritmo rápido de crescimento e de cenários desiguais como o citado, anteriormente, proporcionam uma série de dificuldades para as cidades, tendo em vista que algumas funções fundamentais se tornam de difícil execução, tais como a gestão de resíduos, saúde pública, segurança pública, mobilidade urbana, habitação (Batagan, 2011).

Nesse sentido, a reflexão sobre as cidades e o conceito de cidades inteligentes e sustentáveis, isto é, aquelas que a partir de ferramentas contemporâneas conseguem manter o equilíbrio entre urbanização, desenvolvimento socioeconômico e mitigação dos efeitos adversos ao meio ambiente, faz-se de extrema importância para que seja possível garantir a perpetuação das civilizações (Saraiva et al., 2019).

Dentre os aspectos que o conceito de cidade inteligente e sustentável engloba, a gestão de resíduos sólidos tem sido um dos mais desafiadores devido o volume de resíduos gerados, bem como o potencial nocivo que a gestão inadequada desses resíduos apresenta na saúde pública e ao meio ambiente. Segundo Zago \& Barros (2019), a disposição final dos Resíduos Sólidos Urbanos (RSU) é uma problemática ambiental dos centros urbanos, em especial no Brasil, onde ainda é comum a utilização de lixões ou vazadouros a céu aberto. Para solucionar tal realidade, as cidades necessitam se reinventar e buscar adaptações de modo inteligente e sustentável, no tocante a gestão dos resíduos sólidos (Misra et al., 2018). Isso demanda abordagens baseadas em novos conhecimentos e ferramentas que promovam o planejamento e a adequação da gestão dos resíduos visando atender as demandas e os problemas da urbanização contemporânea (Alqahtani et al., 2020).

Cada etapa gerenciamento de RSU; coleta, transporte, reciclagem, aproveitamento energético, disposição final; vem passando por evoluções tecnológicas com o advento da Indústria $4.0 \mathrm{ou}$ Quarta Revolução Industrial (Logan et al., 2019). Segundo Simão et al. (2019), por volta de 2010, começam a surgir discussões sobre a Indústria 4.0, marcada pela informatização digital, onde a tecnologia da informação e da comunicação se torna integrada aos processos industriais, e as decisões são tomadas de maneira automática, por inteligência artificial, a partir do uso de dados armazenados em nuvem.

A utilização de tecnologia como a Internet das Coisas (IoT) tem se destacado como forma de gerenciar dados e transformá-los em um sistema conectado e inteligente para gestão de RSU (Misra et al., 2018; Wen et al., 2018; Idwan et al., 2019; Alqahtani et al., 2020; Kang et al., 2020; Sharma et al., 2020). A IoT é um conceito relacionado à interconexão por meio digital de objetos cotidianos com a internet, possuindo um potencial imenso de aplicação na gestão de RSU (Lacerda \& LimaMarques, 2015; Nizetic et al., 2020), entre algumas das muitas possibilidades, têm-se: lixeiras inteligentes de coleta de resíduos (etiquetas de Identificação por Radiofrequência - RFID, lixeiras autocompactáveis, detecção de nível de preenchimento de lixeira, segregação automática de resíduos); veículo com sistema de rastreamento; determinação de rota ideal usando Global System for Mobile Communication (GSM), Global Positioning System (GPS) ou Geographic Information System (GIS); robótica automatizada para reciclagem; monitoramento controle de unidades de tratamento de resíduos em tempo real; monitoramento de emissões de gás em aterros (Misra et al., 2018; Ismail et al., 2019; Logan et al., 2019). No entanto, a exploração da aplicação dessas tecnologias ainda está em fase incipiente no gerenciamento de resíduos sólidos.

Diante deste contexto, é evidente a necessidade de analisar o nível atual de conhecimento sobre a temática de Internet das Coisas, aplicada ao gerenciamento de resíduos sólidos, a fim de reunir experiências das pesquisas desenvolvidas, buscando auxiliar gestores municipais na adoção de estratégias eficientes.

As revisões literárias, como os estudos bibliométricos e as revisões sistemáticas, são apontadas por Galvão \& Pereira (2014) como metodologias capazes de esclarecer os avanços científicos e identificar as controvérsias existentes. Os autores relatam, ainda, que este tipo de 
investigação, quando focada em uma questão bem definida, possibilita a identificação, a seleção, a avaliação e a sintetização das evidências relevantes disponíveis na literatura.

Assim, o estudo objetivou realizar uma investigação bibliográfica com relação a aplicação de tecnologias baseadas em Internet das Coisas na gestão de resíduos sólidos, a partir de um modelo híbrido de análise de literatura, utilizando os parâmetros bibliométricos para discussão qualitativa e quantitativa da produção científica e o processo metodológico de revisão sistemática para a síntese de resultados.

\section{Material e Métodos}

Neste estudo foi aplicado um modelo híbrido por meio da sistematização de um conjunto de fases e de etapas, buscando investigar o panorama, as aplicações e os resultados obtidos, seguindo Pluye \& Hong (2014).

Assim, a investigação foi dividida em quatro fases, que delimitaram o método de sistemático da pesquisa: (1) Busca na base de dados, (2) Meta-análise, (3) Análise Bibliométrica e (4) Análise Sistemática (Figura 1).

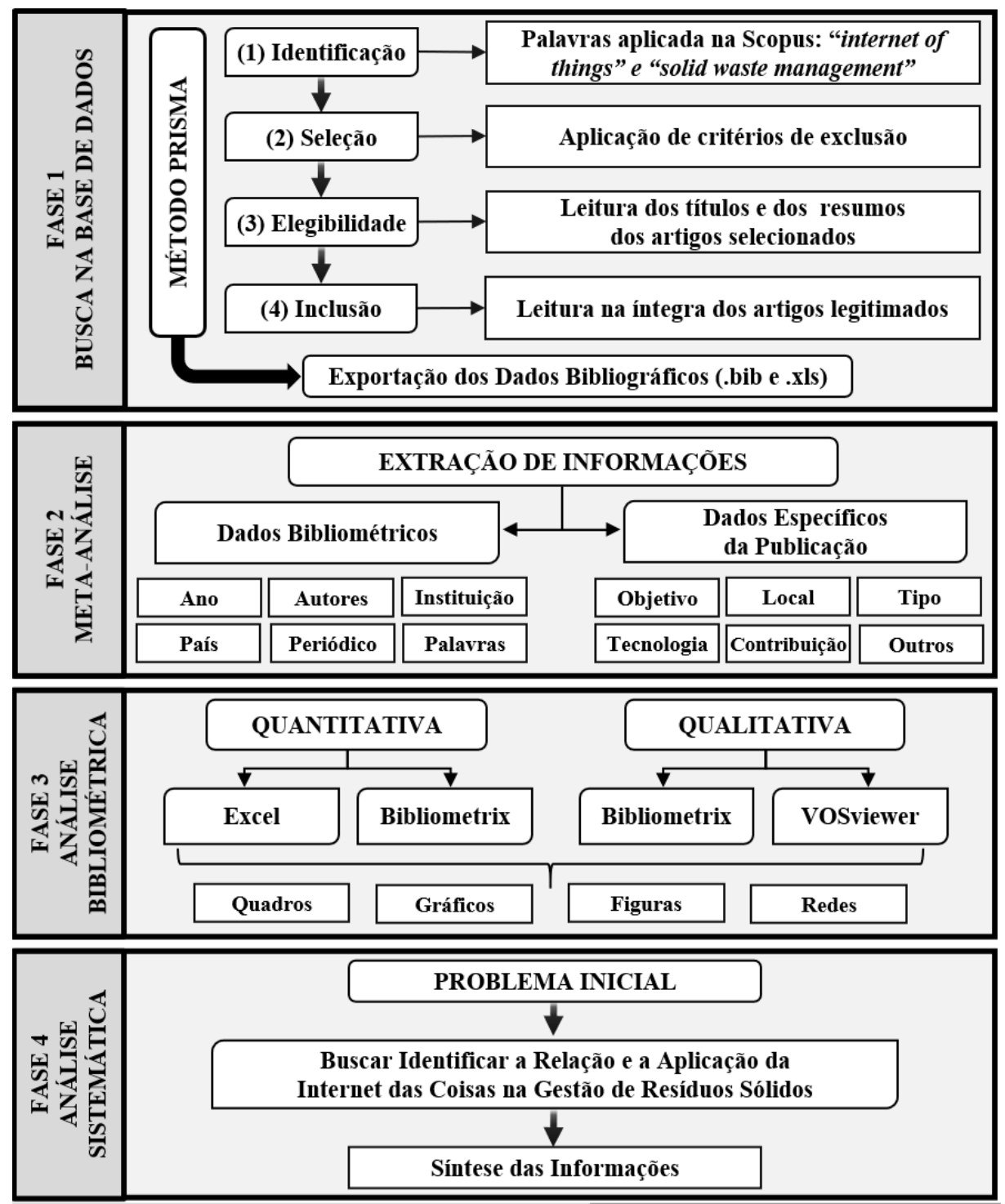

Figura 1. Fluxograma sistemático da pesquisa bibliográfica sobre Internet of Things (IoT). Fonte: Aragão Júnior \& Oliveira Júnior (2021).

\section{Busca na base de dados}

Para este estudo, utilizou-se a base de dados Scopus, plataforma de ampla abrangência científica, por meio do portal da Coordenação de Aperfeiçoamento de Pessoal de Nível Superior (CAPES), com vista a encontrar estudos publicados sobre a IoT aplicada a gestão de resíduos sólidos. A definição dos artigos científicos foi realizada por meio da sistematização da metodologia conhecida como os Principais Itens para Relatar Revisões Sistemáticas e MetaAnálises (PRISMA), que é dividida em quatro etapas: Identificação, Seleção, Elegibilidade e Inclusão (Moher et al., 2010). 
A busca foi realizada no mês de maio de 2020. Na primeira etapa, identificação, buscou-se, simultaneamente, as palavras-chave "internet of things" e "solid waste management" com o operador Booleano "and", resultando na localização de 91 publicações. Depois, na segunda etapa, seleção, foram aplicados critérios de exclusão na amostra inicial, por meio de filtros, ficando somente os artigos científicos de pesquisas e de revisões de literatura, publicados na versão final e em periódicos. Com isso, a amostra foi reduzida para 32 artigos científicos.

Posteriormente, na terceira etapa, elegibilidade, foram realizadas as leituras dos títulos e dos resumos dos artigos selecionados na etapa anterior, resultando na inelegibilidade de dois artigos científicos, porque não tratavam das questões investigadas. Assim, prosseguiu-se com uma amostra de 30 artigos. Na última etapa, inclusão, foram lidos os artigos na íntegra, com vista a excluir os estudos que não faziam parte do eixo temático central analisado, o que tornou a amostra mais precisa, culminando na retirada de dois artigos, pois não estavam dentro do foco abordado. Por fim, incluíram-se 28 artigos científicos para análise da produção científica, exportando os dados bibliométricos desses artigos nos formatos (.bib e .xls) lidos pelos softwares de bibliometria.

A diferença entre a quantidade de documentos localizados e de artigos incluídos na análise evidência a relevância da metodologia PRISMA para a produção de revisão sistemática da literatura, bem como para as análises por meio de indicadores bibliométricos, pois permite a minimização do surgimento de vieses.

\section{Meta-análise}

Os dados bibliométricos exportados dos artigos científicos selecionados foram separados em duas tipologias para utilização nas fases posteriores: dados bibliométricos da publicação e dados específicos da publicação. Como dados bibliométricos da publicação para fase da Análise Bibliométrica, compreenderam-se os itens: ano de publicação do estudo; autores; instituição dos autores; país de origem dos autores; tipo do artigo (pesquisa ou revisão bibliográfica); periódico de publicação; e palavras presentes no título, no resumo e nas palavras-chave. No que tange os dados específicos da publicação para fase da Análise Sistemática, esses dizem respeito às seguintes informações, atreladas ao estudo realizado na pesquisa dos artigos: objetivo da pesquisa; local de investigação; tipo de resíduos sólidos; tecnologias aplicadas para auxiliar a gestão inteligente; e principais contribuições ao estado da arte e/ou às políticas ambientais para o gerenciamento de resíduos sólidos.

\section{Análise bibliométrica}

De acordo com Najmi et al. (2017) a análise bibliométrica apresenta um cenário mais abrangente da literatura, assim como a evolução e o desenvolvimento do tema estudado. A bibliometria foi realizada através de indicadores quantitativa e qualitativa. Os programas Microsoft Office Excel e R Studio (pacote Bibliometrix) foram utilizados para a investigação quantitativa da amostra, por meio de estatística descritiva para confecção de elementos gráficos que explanassem as informações extraídas.

O software VOSviewer foi utilizado para análise qualitativa, a partir da construção de redes bibliométricas, onde as cores indicam os grupos formados (clusters) e o tamanho, os arcos da rede representam a relação entre os elementos interligados e o tamanho do círculo de cada elemento indica o seu impacto na análise realizada. Assim, foram elaboradas redes com base em relações de coautoria (para mapeamento de autores, organizações e países, bem como das relações estabelecidas entre estes, a fim de compreender a estrutura social de uma área de pesquisa), de citação (para mapeamento dos artigos, periódicos, autores e organizações mais citados, buscando entender a influência e a relevância dos estudos para área de pesquisa) e de coocorrência (para mapeamento das palavras presentes nos títulos, nos resumos e nas palavraschave dos artigos e das coocorrências destas palavras nos textos, procurando analisar quais palavras são mais usadas e os conjuntos formados).

\section{Análise sistemática}

A partir do problema inicial: buscar identificar a relação e a aplicação da IoT na gestão de resíduos sólidos, foi realizada a análise dos artigos por meio dos dados extraídos dos artigos. Assim, foram elaboradas tabelas resumos a fim de sintetizar as informações do tipo de resíduos sólidos, das tecnologias aplicadas e das principais contribuições dos estudos, contribuindo para a formulação de estudos futuros e para nortear gestores públicos na aplicação de procedimentos que auxiliam na gestão inteligente de resíduos.

A análise sistemática de literatura permite construir um panorama amplo e consistente do estado da arte, conseguindo localizar as lacunas e as tendências das produções científicas da área estudada, pois consiste na adoção de um método científico que elimina o surgimento de vieses, além de ser passível de reprodução (Liu et al., 2019; Pantaleão \& Veiga, 2019). 


\section{Resultados}

\section{Análise bibliométrica}

Os artigos científicos no âmbito mundial, abordando o tema "Internet das Coisas na Gestão de Resíduos Sólidos" apresentam, até o momento da busca na base de dados Scopus, um total de 28 artigos (Figura 2). Como o primeiro manuscrito data do ano de 2013, há o indício de que o tema analisado vem sendo estudado há pouco tempo.

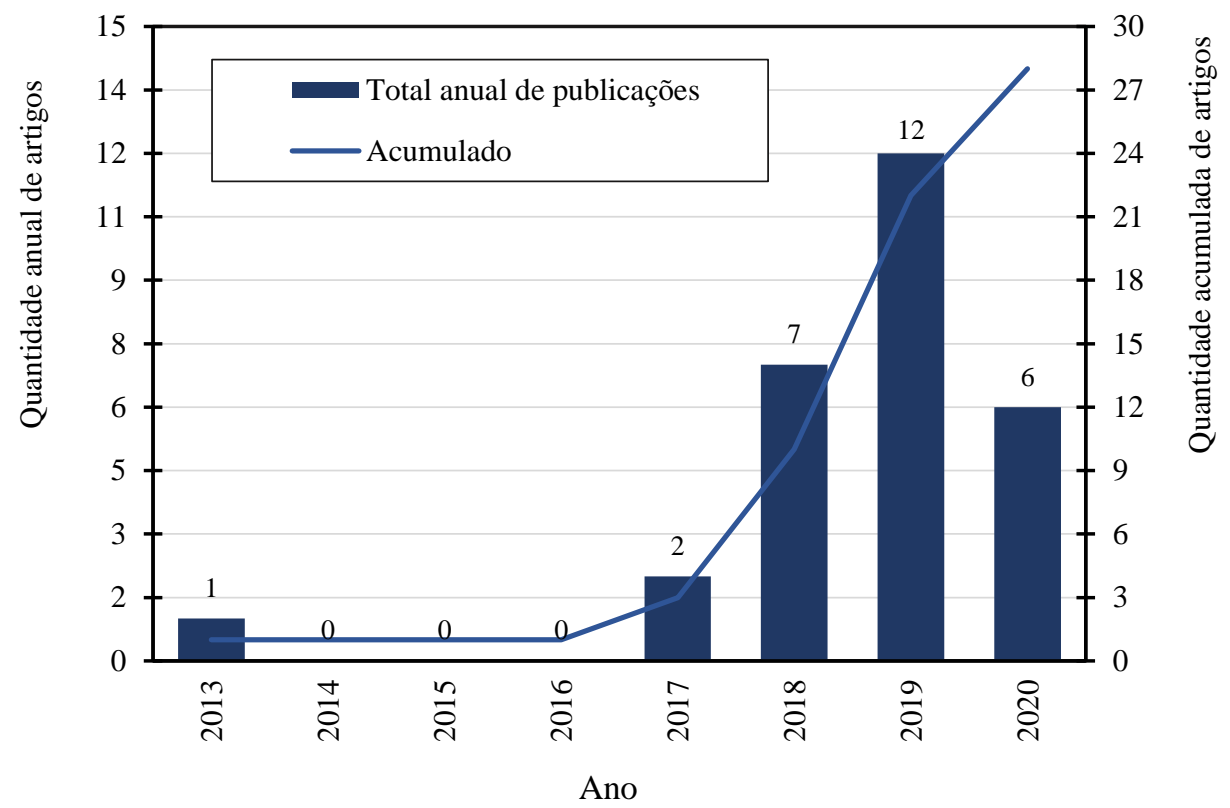

Figura 2. Evolução do número de publicações sobre Internet of Things (IoT), ao longo dos anos. Fonte: Aragão Júnior \& Oliveira Júnior (2021).

O tema abordado está atrelado a novas perspectivas marcadas pela transição da $3^{\text {a }}$ Revolução Industrial para a $4^{\text {a }}$ Revolução Industrial, dando origem a Indústria 4.0. Assim, percebe-se uma crescente quantidade de publicações, oriunda das pesquisas iniciadas na primeira década do século XXI (GAŠOVÁ et al., 2017). Nota-se que após 2013, alguns anos não tiveram publicações, foram eles: 2014, 2015 e 2016. Contudo, ficou evidente que o número de publicações vem aumentando ao longo dos anos, principalmente a partir de 2018, existindo uma notória tendência de crescimento, visto que o número de publicações nos últimos três anos corresponde a, aproximadamente, $90 \%$ dos artigos encontrados, evidenciando que a área de estudo se encontra em ascensão. Destaca-se que a quantidade dos artigos referentes a 2020 não representa a totalidade, pois a busca foi realizada no mês de maio, logo, a redução do número de artigos publicados no ano 2020 em comparado com 2019, não indica uma redução no interesse de estudo no tema.

$\mathrm{O}$ artigo seminal (estudo mais antigo que compõe a base de dados) dessa pesquisa, publicado por $\mathrm{Lu}$ et al. (2013), discutiu a aplicação de tecnologia da informação para auxiliar no processo de gerenciamento integrado de resíduos sólidos urbanos e de resíduos perigosos. Os autores apresentam uma visão holística e uma discussão aprofundada da informática ambiental aplicada ao gerenciamento de resíduos sólidos e perigosos, apontando tendências futuras com o objetivo de melhorar a gestão, a exemplo de sistemas de banco de dados, da aplicação do GIS, de mecanismos de inteligência artificial no apoio a tomadas de decisão, de sensoriamento remoto, dentre outras.

Considerando os 135 autores dos 28 artigos, percebe-se que, apenas 10 países, possuem dois ou mais autores, totalizando cerca de $41 \%$ dos autores: Índia com 22, China com 10, Malásia com cinco, Estados Unidos com quatro, Espanha e Grécia com três e Austrália, Brasil, Jordânia e Reino Unido com dois. Ainda, considerando os 10 países que têm dois ou mais autores, nota-se que o continente asiático foi o que teve maior quantidade de autores pesquisando a temática, com 39 autores, correspondendo a aproximadamente $71 \%$, seguido do continente europeu, com 8 autores (14\%), o continente americano, com 6 (11\%), e o continente oceânico, com 2 (4\%).

Vale destacar que o continente africano não teve nenhum autor, o que é preocupante tendo em vista que esse continente se concentra um número elevado de países em condição de subdesenvolvimento ou emergentes, enfrentando problemas com a gestão de resíduos sólidos e seus impactos negativos ao meio ambiente (Couth \& 
Trois, 2012). Além disso, esse resultado é coeso com os dados que apontam para liderança dos países asiáticos na corrida científica e tecnológica mundial, alcançada através de investimentos consideráveis em pesquisa e em desenvolvimento, proporcionando, com isso, o estímulo por estudos tecnológicos que contribuem para o avanço da Indústria 4.0 e culminam para as profundas alterações nos padrões de produção, de gestão e de distribuição, colaborando para construção de cidades inteligentes e sustentáveis (Diniz, 2019).

Os 135 autores dos 28 escritos estão distribuídos em diversas instituições, apenas 9 afiliações detêm mais de dois pesquisadores, totalizando 21 autores. As outras instituições possuem somente um autor (Tabela 1).

Tabela 1. Principais instituições com publicações sobre Internet of Things (IoT). Fonte: Aragão Júnior \& Oliveira Júnior (2021).

\begin{tabular}{lcc}
\hline Instituições & País & Número de autores \\
\hline Universidad de Castilla-La Mancha & Espanha & 3 \\
Universiti Teknologi Malaysia & Malásia & 3 \\
University of West Attica & Grécia & 3 \\
Beijing University of Technology & China & 2 \\
Bharathiar University & Índia & 2 \\
Itmo University & Rússia & 2 \\
Koneru Lakshmaiah Education Foundation & Índia & 2 \\
Monash University Malaysia & Malásia & 2 \\
Tsinghua University & China & 2 \\
\hline
\end{tabular}

Desse modo, as afiliações dos autores reiteram a elevada produção do continente asiático, uma vez que dentre as nove instituições com mais autores, seis são da Ásia. Em seguida, destaca-se o continente europeu com três instituições. É importante salientar que continentes como a América e a África, que possuem países subdesenvolvidos ou emergentes, consequentemente, apresentam problemas urbanos relacionados com a gestão de resíduos sólidos, não aparecem nesta lista. Isso aponta para caminhos de possíveis parcerias entre os grupos consolidados com a temática e os grupos dos continentes americano e africano que ainda não desenvolvem pesquisas no tema, de modo a maximizar em cidades emergentes os efeitos da gestão de resíduos sólidos baseados em tecnologias como a Internet das Coisas.

Segundo Van Eck \& Waltman (2010), por meio da análise de redes de coautoria é possível identificar como pesquisadores, instituições de pesquisa ou países se relacionam de acordo com a quantidade de estudos que realizam e publicam em cooperação estabelecida. Com isso, a partir do software VOSviewer, foi realizada a análise de coautorias dos países correspondentes as instituições que os autores dos artigos fazem parte, permitindo compreender as relações estabelecidas. Cada nó representa um país, e os tamanhos dos nós indicam o número de artigos publicados. O link que conecta dois nós representa a relação de cooperação entre dois países, e a espessura do link indica a intensidade da cooperação. Assim, para mapear as principais redes de coautoria, foram considerados apenas os países com pelo menos dois documentos na amostra. Tal restrição produziu uma rede com sete nós (países), distribuídos em três clusters de colaboração em pesquisa (Figura 3).

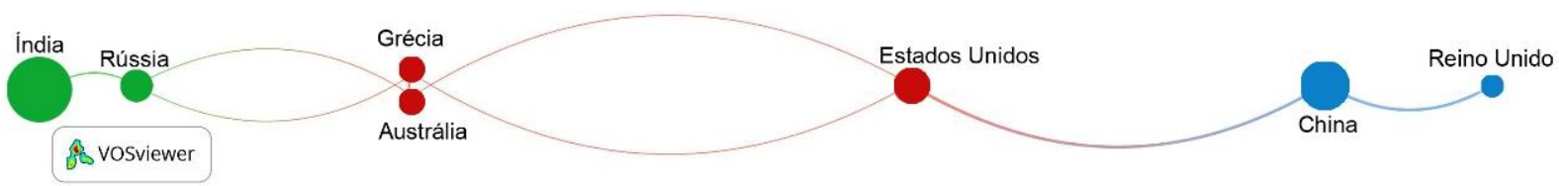

Figura 3. Rede de coautoria em artigos sobre Internet of Things (IoT), entre os países. Fonte: Aragão Júnior \& Oliveira Júnior, utilizando o VOSviewer versão 1.6.14 (2021).

A linearização da rede de coautoria evidência a baixa interação entre instituições de países diferentes e a dispersão das pesquisas do campo de estudo. Apenas 11 artigos tiveram a elaboração por autores de países diferentes: Lu et al. (2013), Gu et al. (2017), Bong et al. (2018), Wen et al. (2018), Shah et al. (2018), Anagnostopoulos et al. (2018), Pardini et al. (2019), Xue et al. (2019), Logan et al. (2019), Idwan et al. (2019) e Velvizhi et al. (2020). Destaca-se a formação de três grupos principais de colaboração: Índia e Rússia (clusters em cor verde); Estados Unidos, Grécia e Austrália (clusters em cor vermelha); e China e Reino Unido (clusters em cor azul). Além disso, percebe-se 
também secundariamente a cooperação entre Rússia, Grécia e Austrália; Grécia e Austrália; e Estados Unidos e China.

Diante disso, depreende-se que existiu uma pequena colaboração científica entre grupos de pesquisa de países distintos, como também é possível afirmar que há uma baixa cooperação entre os integrantes de grupos do mesmo país. Essas verificações vão de encontro com algumas das finalidades que norteiam a essência de um grupo de pesquisa: apoio e partilha de experiências entre os membros e busca por parcerias entre grupos de outras instituições (Katz \& Martins, 1997; Newman, 2004), a fim de gerar uma rede de cooperação que acrescente no crescimento do conhecimento técnico-científico, contribuindo para o bem-estar da sociedade e consolidando a "tríplice acadêmica" (ensino, pesquisa e extensão).

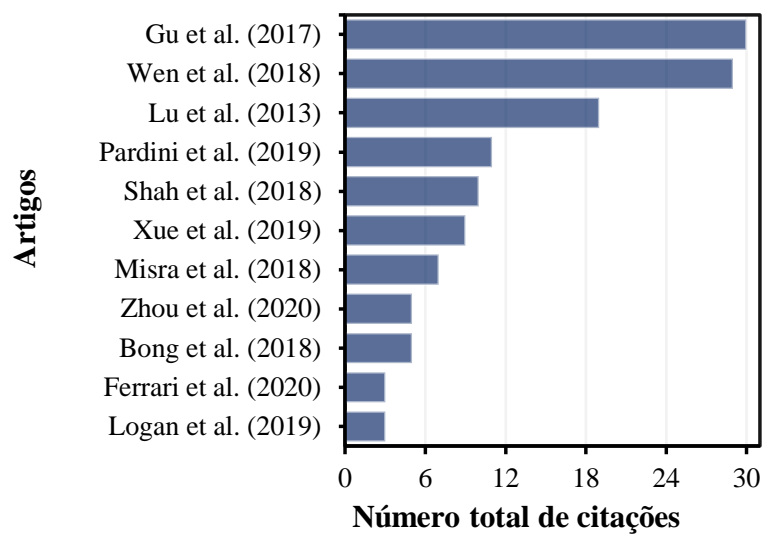

(a)
Conforme a Agência USP de Gestão da Informação Acadêmica (2016), o número de citações depende de diversas variáveis: a pertinência das contribuições do estudo, o periódico de publicação, a base de indexação, o tempo de publicação.

O total de citações dos 28 artigos na base de dados da Scopus foi igual a 140, sendo aproximadamente $94 \%$ referentes a apenas 11 artigos (Figura 4a), apontando para uma provável relevância e/ou embasamento desses artigos para a comunidade técnica-científica. Ao analisar os 11 artigos com as maiores médias de citações por tempo de publicação do estudo (Figura 4b), verifica-se que todos constam nas duas análises, comprovando a importância desses artigos e dos seus correspondentes autores para o tema abordado nesse estudo.

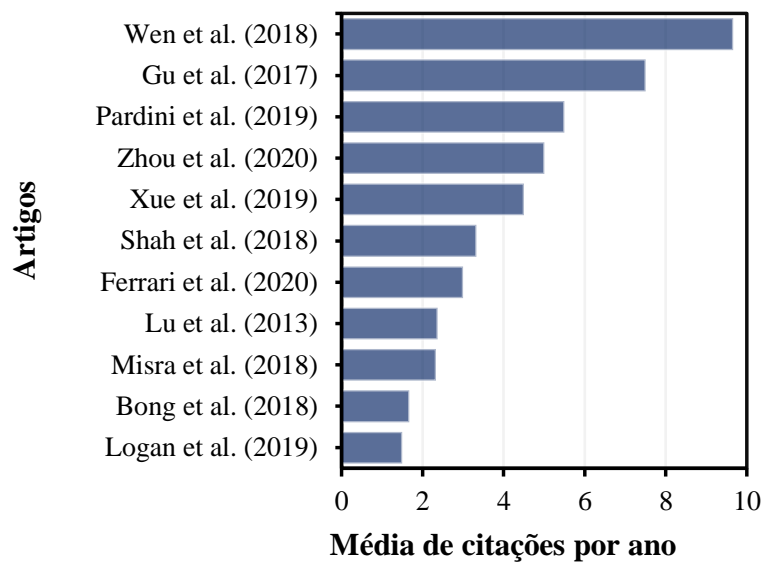

(b)

Figura 4. Análise das citações dos artigos sobre Internet of Things (IoT): (a). 11 artigos com maior número de citações; (b). 11 artigos com maiores médias de citações por tempo de publicação. Fonte: Aragão Júnior \& Oliveira Júnior (2021).

Considerando esses artigos amplamente citados categoricamente pelo banco de dados da Scopus, obtiveram destaque com maior contribuição nas duas categorias analisada: $\mathrm{Gu}$ et al. (2017) e Wen et al. (2018). Os dois artigos abordaram a aplicação de sistemas inteligentes combinados a IoT e a Big Data para a gestão de resíduos de equipamentos elétricos e eletrônicos (REEE) e para a gestão de resíduos alimentares de restaurantes. É importante ressaltar que o primeiro artigo publicado sobre a temática; Lu et al. (2013), está em terceiro lugar na lista dos artigos mais citados, no entanto, aparece na oitava posição na lista dos artigos com maiores médias de citações por ano. Assim, existe a possibilidade de inicialmente Lu et al. (2013) terem influenciado significativamente a produção científica, servindo de apoio para pesquisas posteriores a publicação do estudo, confirmando o impacto temporário dos artigos, tendo em vista a evolução técnicocientífica. Ademais, pôde-se confirmar um dos aspectos que interferem no número de citações, o tempo de publicação do manuscrito, visto que, dos 18 artigos datados de 2019 e de 2020, somente cinco; Logan et al. (2019), Pardini et al. (2019), Xue et al. (2019), Ferrari et al. (2020) e Zhou et al. (2020) apareceram na lista dos 11 artigos com maiores números de citações e com maiores médias de citações, por tempo de publicação.

Buscando entender a sinergia de citações entre os 28 artigos incluídos nesta pesquisa, foi elaborada a rede de citação entre os artigos analisados por meio do software VOSviewer, onde o tamanho do nó indica a quantidade total de citação que o artigo recebeu e a ligação entre os nós representa a ocorrência de citação (Figura 5). 


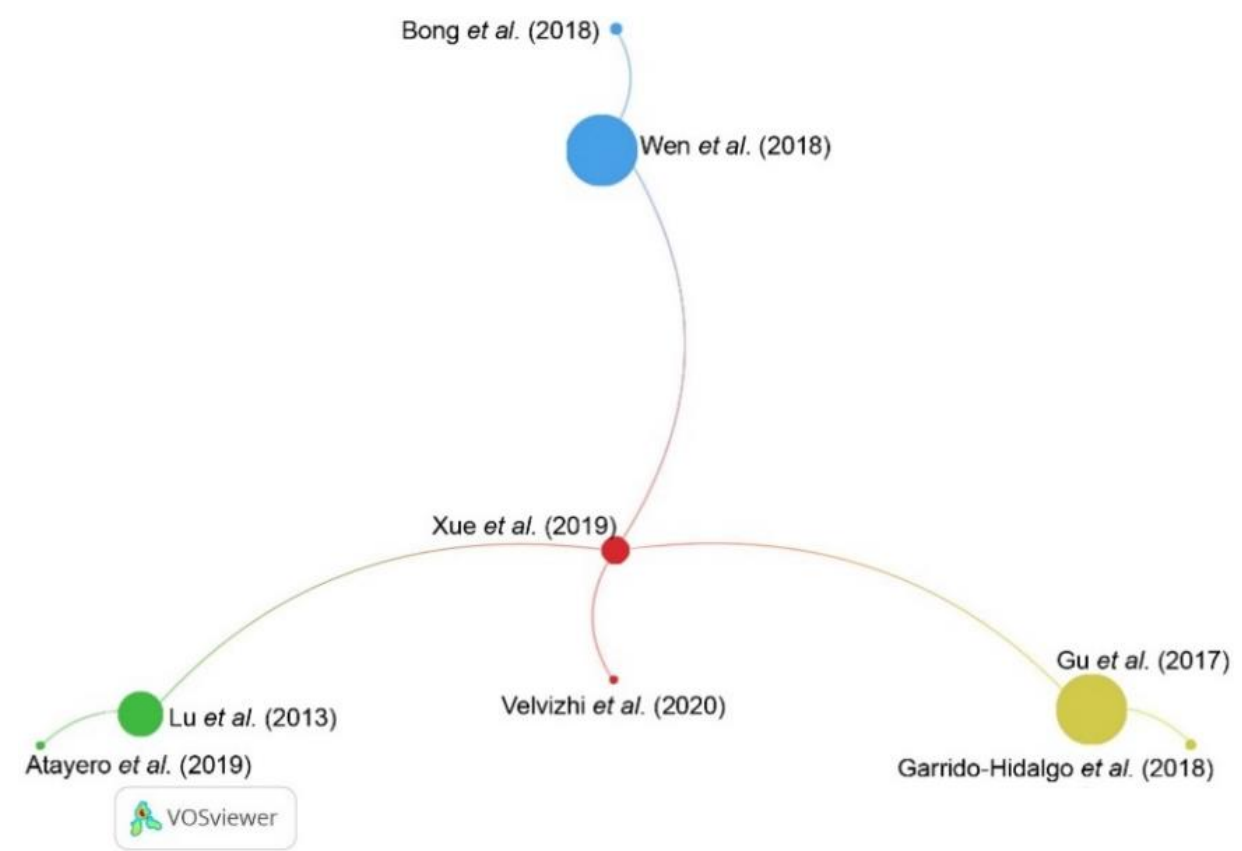

Figura 5. Rede de citação entre os artigos sobre Internet of Things (IoT). Fonte: Aragão Júnior \& Oliveira Júnior, utilizando o VOSviewer versão 1.6.14 (2021).

A rede de citação dos artigos foi formada por oito nós, organizados em quatro clusters. No cluster vermelho, contendo dois nós, representa que Velvizhi et al. (2020) citou o estudo de Xue et al. (2019), que, por sua vez, citou os três artigos com maior quantidade de citações: Wen et al. (2018) - cluster azul, Gu et al. (2017) - cluster amarelo, e Lu et al. (2013) - cluster verde; sendo esses, respectivamente, também citados nos estudos de Bong et al. (2018), de Garrido-Hidalgo et al. (2018) e de Atayero et al. (2019). Essas citações demostram a importância desses artigos para a amostra analisada, bem como uma proximidade entre esses. Os 20 artigos restantes não citaram e nem receberam citação dos artigos que fazem parte desse estudo.

A relação de coocorrência de palavras é determinada pela quantidade de aparição conjuntamente dos vocábulos em diversos campos dos artigos pertencentes a base de documentos analisada, podendo ser nas palavras do título, do resumo, da lista de palavras-chave dos autores ou das revistas (Van Eck \& Waltman, 2017). Segundo o princípio da Lei de Zipf, com a frequência de reprodução das palavras nos textos é possível gerar uma lista ordenada de termos predominantes que determinam a temática central de um conjunto de documentos (Machado Junior et al., 2016). Ao analisar essas redes, onde tamanho do nó indica a frequência de ocorrência da palavra e a relação entre os nós é tão mais forte quanto maior a proximidade entre eles, é possível mapear possíveis temáticas de pesquisa que ainda não foram muito exploradas ou mesmo novas vertentes de estudos.

A primeira análise foi da rede de coocorrência das palavras presentes nos títulos e nos resumos dos artigos incluídos na pesquisa. Através da utilização do software VOSviewer, foi encontrado um total de 1104 palavras, logo, para facilitar a visualização, restringiu a formação da rede com palavras que tivessem no mínimo 5 ocorrências (Figura 6).

A rede de coocorrência das palavras dos títulos e dos resumos foi formada por 28 nós, organizados em quatro clusters. No cluster vermelho, contendo 11 nós, nota-se que aqueles com maior frequência de ocorrência são bin (lixeira), level (nível), information (informação), time (tempo), cloud (nuvem) e smart city (cidade inteligente). Esse conjunto de palavras sugere pesquisas que abordam a questão da automatização de lixeiras a partir de sensores que monitoram o nível de preenchimento, compartilhando informações na nuvem, ou seja, de forma online ou em rede, para a coleta e a substituição dessas lixeiras inteligentes. Esses estudos contribuem para a criação de uma cidade inteligente, marcada pela otimização a partir de sensores eletrônicos que coletam dados usados para auxiliar na gestão e na tomada de decisão, aumentando a eficiência da utilização de recursos naturais e financeiros. 


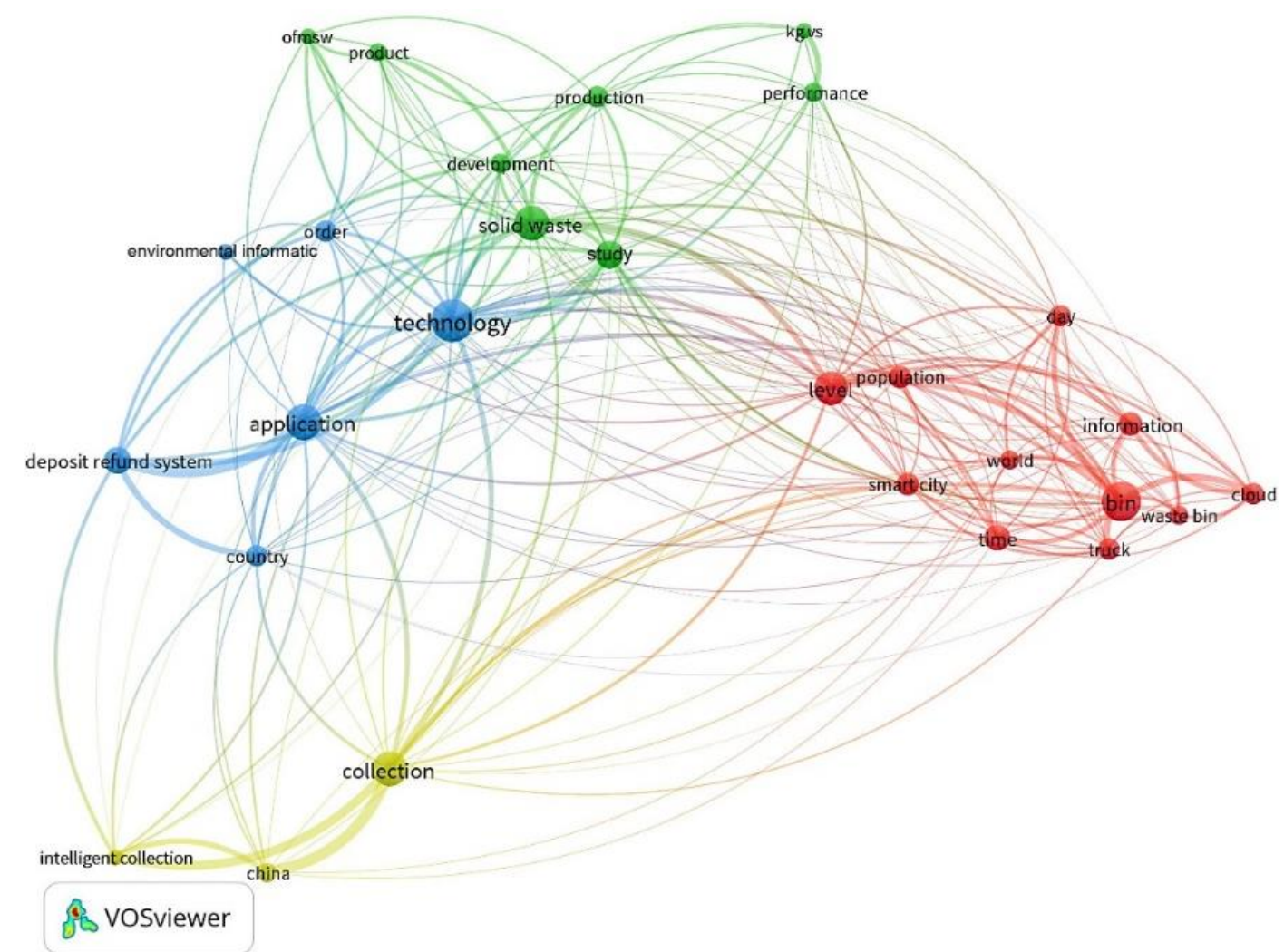

Figura 6. Rede de coocorrência das palavras presentes nos títulos e nos resumos de artigos sobre Internet of Things (IoT). Fonte: Aragão Júnior \& Oliveira Júnior, utilizando o VOSviewer versão 1.6.14 (2021).

Entre os oito nós agrupados no cluster verde, destacam-se, nessa ordem, solid waste (resíduos sólidos), study (estudo), development (desenvolvimento) e production (produção). Essas ocorrências estão relacionadas a estudo que utilizam a IoT na gestão de resíduos sólidos a fim de auxiliar no desenvolvimento e na produção de sistemas inteligentes de gerenciamento de resíduos que proporcionem economias substanciais em tempo, custo, duração da viagem e utilização de veículos. O terceiro cluster, azul, reuniu seis palavras, sendo as mais frequentes technology (tecnologia), application (aplicação) e deposit refund system (sistema de reembolso de depósitos). A linha de pesquisa que tais palavras sugerem foi desenvolvida por Zhou et al. (2020) que analisaram a prática do sistema de reembolso de depósitos de embalagens de bebidas (latas, garrafas PET, garrafas de outro tipo de plástico, garrafa de vidro e caixas Tetra Pak) em 40 países ou regiões, contribuindo para fornecer suporte para implementação de políticas públicas que incentivem a logística reversa para a consolidação da economia circular.

O último aglomerado de palavras, cluster amarelo, possui três nós, sendo as palavras mais destacadas collection (coleção) e China. Esses termos estão atrelados ao estudos realizado por Xue et al. (2019), abordando um caso da China, que realiza uma análise comparativa entre a coleta inteligente usando IoT, por meio da interação homem-máquina, e o a coleta informal de RSU, a partir da interação humano-humano, avaliando aspectos econômicos e sociais inerentes aos dois modelos. Os autores relatam vantagens da coleta inteligente em relação a coleta informal, de modo que essas vantagens tornam a coleta inteligente com o potencial de integrar ou mesmo substituir a coleta informal.

A segunda análise foi da rede de coocorrência das palavras presentes nas palavraschave determinadas pelos autores Foram encontradas 372 palavras, assim, para facilitar a visualização, restringiu a formação da rede com palavras que tivessem pelo menos três ocorrências (Figura 7).

A rede de coocorrência das palavras das palavras-chave dos autores foi formada por 23 nós, organizados em três clusters. No cluster vermelho, contendo nove nós, nota-se que aqueles com maior frequência de ocorrência são Internet of Things IoT (Internet das Coisas), waste management (gestão de resíduos) e smart city (cidade inteligente). Esse conjunto de palavras está alinhado com as palavras-chave aplicadas inicialmente na pesquisa para localizar os documentos. Com isso, percebe-se a utilização da IoT na gestão de resíduos buscando a construção de uma cidade inteligente. 


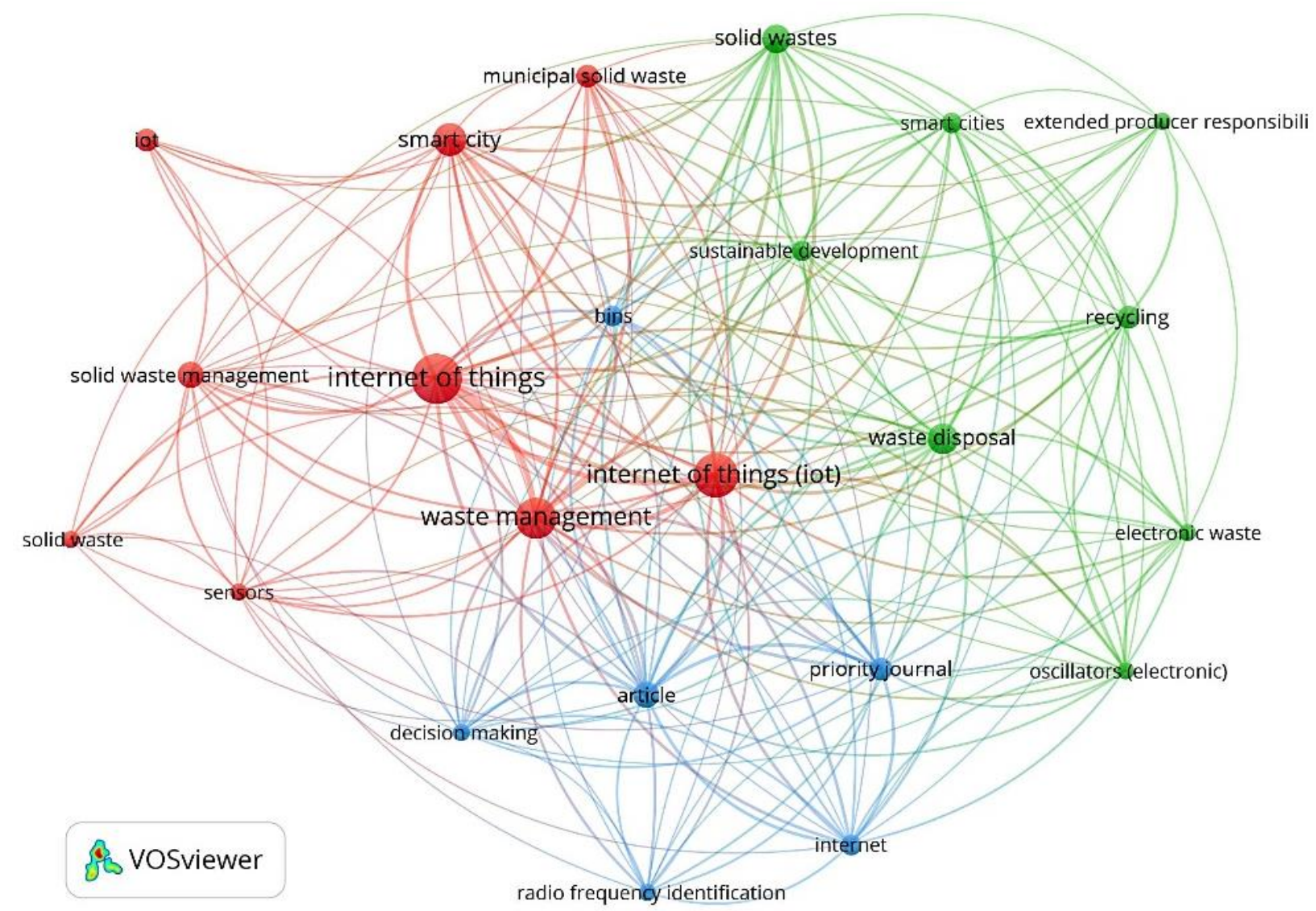

Figura 7. Rede de coocorrência das palavras-chave utilizadas pelos autores em estudos sobre Internet of Things (IoT). Fonte: Aragão Júnior \& Oliveira Júnior, utilizando o VOSviewer versão 1.6.14 (2021).

Entre os oito nós agrupados no cluster verde, destacam-se, sequencialmente, solid wastes (resíduos sólidos), waste disposal (depósito de lixo), recycling (reciclando) e sustainable development (desenvolvimento sustentável). Essas ocorrências destacam os benefícios dos sistemas inteligentes para consolidação da reciclagem, colaborando para o desenvolvimento sustentável das cidades. O último cluster, azul, reuniu seis palavras. Esses termos associam as lixeiras (bins) a tomada de decisão (decision making), internet e RFID, demostrando que alguns estudos se referem a otimização de lixeiras para envio de informações, por meio da internet ou da radiofrequência, que auxiliam na tomada de decisão.

\section{Revisão sistemática}

A Internet das Coisas desponta com uma das tecnologias mais importante no mundo moderno, ganhando rapidamente um novo paradigma nas telecomunicações sem fio nas áreas de Economia Inteligente, Mobilidade Inteligente, Ambiente Inteligente, Vida Inteligente e Governança Inteligente (Velvizhi et al., 2020). Com isso, tornou-se um meio de interação humanohumano e humano-computador, integrando pessoas, máquinas digitais e dispositivos de computação.

A IoT pode ser vista como a combinação de diversas tecnologias, complementadas buscando viabilizar a integração dos objetos no ambiente físico ao mundo virtual (Figura 8), sendo usada para o progresso do hardware nos dispositivos e para armazenar os dados, através da nuvem. Essa conexão entre o hardware nos dispositivos e a nuvem de dados ocorre basicamente em quatro níveis: sensores ou atuadores, sistemas de aquisição de dados, pré-processando e análise de dados (Dhana Shree et al., 2019; Srikant et al., 2019; Velvizhi et al., 2020). 


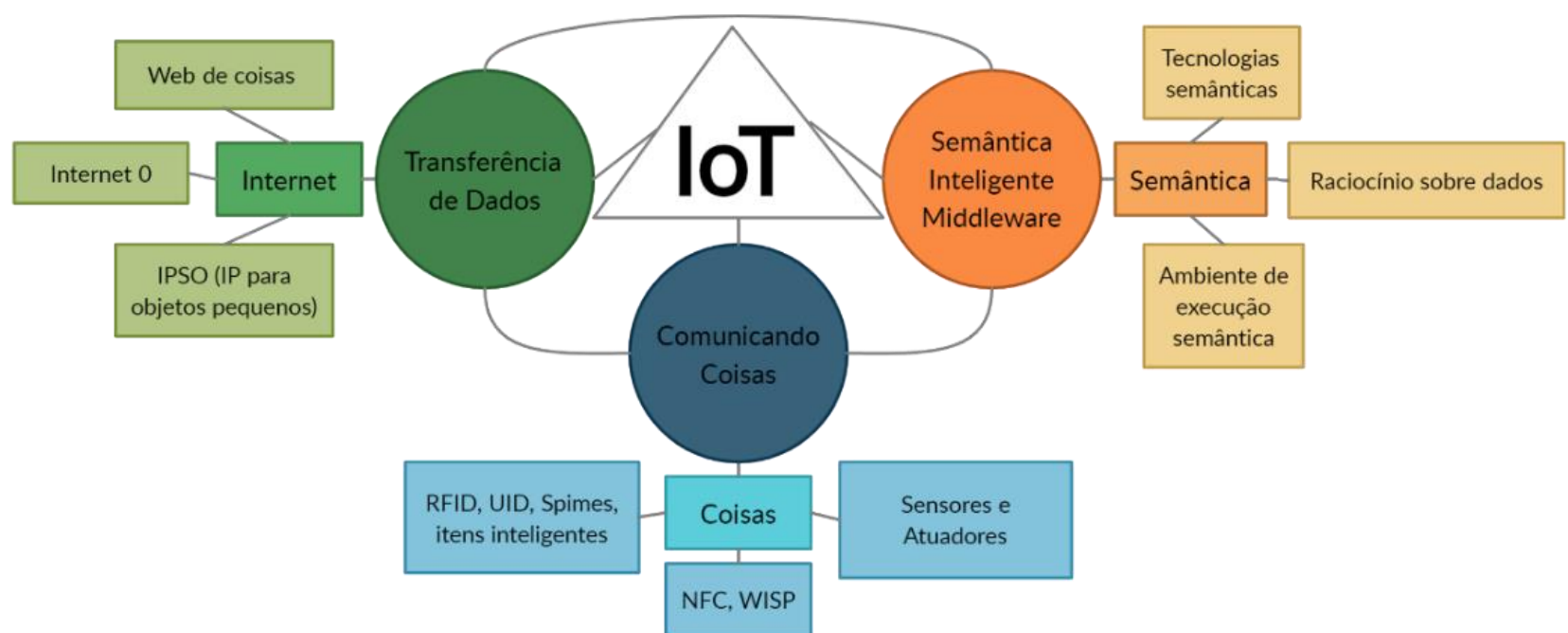

Figura 8. Paradigma da Internet das Coisas (IoT) como resultado da convergência de diferentes visões. Fonte: Adaptado de Velvizhi et al. (2020).

Em linhas gerais, nos sistemas de gestão de resíduos sólidos, a IoT vem sendo aplicada na criação de dispositivos capazes de monitorar o nível e os tipos de resíduos presentes nos coletores como lixeiras e contêineres. Estas informações são enviadas e armazenadas em uma nuvem de onde é possível emitir comandos como acionar um caminhão mais próximo da lixeira/contêiner para realizar a coleta dos resíduos e conduzi-los para uma central de tratamento (Misra et al., 2018; Wen et al., 2018; Idwan et al., 2019; Srikant et al., 2019; Alqahtani et al., 2020; Kang et al., 2020; Sharma et al., 2020).

Os principais dispositivos utilizados no processo de equipar as lixeiras/contêineres são os sensores. $\mathrm{Na}$ atualidade, existem diversos tipos de sensores, dentre eles o mecânico, o ultrassônico, o indutivo, o capacitivo e o fotoelétrico (Rodelas et al., 2020). Estes sensores, por vezes, fazem parte de um sistema de rede sem fio onde os envios e armazenamentos das informações entre o dispositivo e a nuvem de dados são feitas via conexão Wireless Fidelity (Wi-Fi), bluetooth, identificação óptica, dentre outros (Monishan et al., 2019).

Foram reunidos os tipos de resíduos e as principais formas de utilização da tecnologia IoT na gestão dos resíduos sólidos dos artigos selecionados, excluindo os estudos que abordavam revisões de literatura (Tabela 2). Foram elencados 20 artigos, entre eles, dois abordam a utilização da IoT na gestão de REEE e os demais 18 artigos, na gestão de RSU e de resíduos orgânicas oriundos de alimentos. Ainda entre os 20 artigos, tem-se que seis empregam tecnologias do tipo redes sem fio e os 14 demais, empregam sensores.

Tabela 2. Tipos de resíduos e formas de uso da tecnologia Internet of Things (IoT). REEE = Resíduos Eletroeletrônicos; RSU = Resíduos Sólidos Urbanos; Fonte: Aragão Júnior \& Oliveira Júnior (2021).

\section{$\begin{array}{lll}\text { Referência } & \begin{array}{l}\text { Tipo de } \\ \text { resíduos }\end{array} & \text { Tecnologias empregadas nas pesquisas }\end{array}$}

Sensor ultrassônico capaz de determinar a nível da caixa de coleta, com Arduino para fornecer energia e permitir que um

Kang et al. (2020) REEE programa controle o sensor e o módulo $\mathrm{Wi}$-Fi, sendo vinculados a um sistema de banco de dados (Google Firebase) baseado em nuvem

Rodelas et al. RSU Sensores ultrassônicos e algoritmo de programação via Arduino. (2020) Conexão com GPS e com a estação de trabalho via Wi-Fi

Sensor capacitivo para medição da umidade do resíduo orgânico, $\begin{array}{ll}\text { Ferrari et al. (2020) } & \text { Resíduo } \\ \text { Orgânico }\end{array}$ onde o sinal fornecido foi adquirido por meio do conversor analógico-digital integrado no microcontrolador do Arduino e os dados coletados são elaborados de acordo com a curva de calibração para obter a umidade do resíduo

\begin{tabular}{l}
$\begin{array}{l}\text { Garrido-Hidalgo et } \\
\text { al. (2020) }\end{array}$ \\
\hline
\end{tabular}


Idwan et al. (2019) RSU

Dhana Shree et al. (2019)

RSU

Jacob et al. (2019) RSU

$\begin{array}{ll}\text { Logan et al. (2019) } & \text { Resíduo } \\ \text { Orgânico }\end{array}$

\begin{tabular}{ll}
\hline $\begin{array}{l}\text { Monishan et al. } \\
(2019)\end{array}$ & RSU \\
\hline $\begin{array}{l}\text { Chakravarthi et al. } \\
(2019)\end{array}$ & RSU
\end{tabular}

Atayero et al. (2019)

RSU

\begin{tabular}{lll}
\hline Xue et al. (2019) & RSU & P \\
\hline Bhanot et al. (2019) & \multirow{2}{*}{ RSU } & P \\
\hline
\end{tabular}

Anagnostopoulos et al. (2018)

RSU

Shah et al. (2018) RSU

Misra et al. (2018) RSU

Resíduos

Wen et al. (2018)

Alimentares de

Restaurantes

Sensor ultrassônico de alcance (para medir o nível de preenchimento da lixeira) conectado a uma placa Arduino e ao módulo GSM, que é então colocado em cada lixeira, fornecendo um link de comunicação para o centro de controle municipal

Sensor ultrassônico para medir o nível de resíduos, conectado ao módulo GSM/GPS/Bluetooth, estabelecendo a comunicação com a gestão. O sensor térmico para detectar mudança no nível de calor e acionar o servomotor para abrir a tampa

O sensor ultrassônico estima o nível de resíduos, enviando uma mensagem quando atinge o limite, através de Arduino composto por um módulo GSM do aplicativo Android, que possui interfaces e módulo Wi-Fi, fornecendo o caminho mais curto

Sensores para o monitoramento on-line da composição e do volume de biogás produzido através de Controlador Lógico Programável (CLP), monitorando os dados pelo aplicativo Team Viewer, podendo ser gravado e transferido pelo Program Data Logger V130

Lixeiras equipadas com sensor infravermelho, sensor de chuva e sensor que se comunica via Bluetooth e Wi-Fi com a central

Caixote do lixo conectado por Wi-Fi que emite sinal de alerta de preenchimento para estação de trabalho via aplicativo

Contêineres equipados com sensores ultrassônicos, os dados são comunicados via rede Wi-Fi notificando o nível de preenchimento Máquina equipada com sensores para identificação do material Planta de segregação de resíduos por meio de sensores e aplicativo que permite o controle remoto pela internet Criação de um Sistema Multiagente para avaliar a alocação dinâmica de caminhões em relação ao comportamento de lixeira com sensores de preenchimento durante as variações das 4 estações

Elaboração de um modelo de otimização estocástica baseado em programação restrita ao acaso desenvolvido para otimizar o planejamento das operações de coleta de lixo

Sensor ultrassônico para detectar o nível de resíduo na lixeira. As informações são enviadas pela Internet para um servidor sendo usadas para monitorar os caixotes do lixo e auxiliar na tomada de decisão do recolhimento do caixote do lixo

RFID nas lixeiras com informações referentes ao estabelecimento. Caminhão inteligente equipado com leitor RFID, câmera de vigilância, sensor de peso, módulo GPS, módulo General Packet Radio Service (GPRS) e computador de bordo para envio de dados

Sensor ultrassônico para medir o preenchimento da lixeira e Raspberry Pi para processar as informações. Os dados são enviados para a nuvem (ThingSpeak) usando o módulo Wi-Fi do

Gaddam et al. (2018)

RSU lixeira ultrapassa o nível, a localização dos compartimentos e o caminho mais curto são mostradas no aplicativo usando o Google Maps

Sensor infravermelho para detectar o nível de lixo e mau cheiro

Anand et al. (2018) RSU proveniente com acesso remoto e transmitir imediatamente a informação ao município através da IoT, enviando mensagens de alerta para o motorista do caminhão coletor de lixo

Vaisali et al. (2017) RSU Lixeira com sensores de infravermelho para detecção de preenchimento e mau cheiro enviando os dados por GSM 
Dentre os que utilizaram redes sem fios, Rodelas et al. (2020) relatam o desenvolvimento e aplicação de um sistema de redes sem fio aplicado ao gerenciamento de resíduos sólidos urbanos na Filipinas, sendo o mesmo capaz de auxiliar na segregação dos resíduos biodegradáveis e não degradáveis, escolha de rotas otimizadas entre os coletores de resíduos e o aterro, além de armazenar informações de geração de resíduos e localização dos domicílios geradores.

Garrido-Hidalgo et al. (2020) buscaram aplicar conceitos e ferramentas IoT para auxiliar no gerenciamento de REEE que possibilitem a logística reversa dos resíduos e em seguida a inserção dos mesmo em uma nova cadeia de suprimentos visando como filosofia de produção a Economia Circular.

Os artigos de Monishan et al. (2019) e de Atayero et al. (2019) relataram o desenvolvimento de um sistema inteligente para gerenciamento de RSU a partir de automação da caixa que coleta os resíduos que notifica o momento de realizar a coleta dos resíduos para serem conduzidos até a destinação final e além disso o sistema otimiza as rotas e auxilia no transporte dos resíduos da coleta até a destinação final, sendo imprescindível para construção de comunidades inteligentes e conectadas.

Dentre os que utilizaram sensores, Velvizhi et al. (2020) elencaram possibilidades de aplicações para as diferentes frações dos RSU, orgânicos e não orgânicos, dentro de um contexto de economia circular e com auxílio de tecnologias. Os autores sugerem melhor eficiência do processo com a utilização de sensores conectados via internet a um banco de dados para auxiliar na separação, na coleta, no transporte e na destinação.

A partir do aproveitamento e inertização da fração orgânica dos resíduos sólidos urbanos misturados com resina, Ferrari et al. (2020) desenvolveram um novo material para painéis automatizados. Logan et al. (2019) relataram a experiência de um sistema criado para monitorar o processo de digestão anaeróbica de resíduos orgânicos, sendo possível verificar as condições físico químicas durante o processo de biodegradação da massa e a produção de biogás. Ainda em relação aos de resíduos orgânicos, Bong et al. (2018) criaram um sistema inteligente para gerenciamento de resíduos alimentares de restaurantes e elenca pontos positivos e negativos do mesmo. Os autores chamam a atenção de que a ocorrência de divergências entre as agências governamentais da cidade sobre como interpretar dados do sistema de IoT foi uma das razões que levou a algumas ineficiências na gestão, usando o sistema inteligente.

Visando contribuir para o alcance ideológico de agricultura inteligente, Gaddam et al. (2018) desenvolveram um sistema automatizado para auxiliar na coleta, modo e frequência de transporte, desempenho de pré-tratamento e unidades de tratamento e geração dos produtos desejados na cadeia de suprimento agrícola.

Kang et al. (2020) tratam em seu estudo sobre a necessidade de adequar o gerenciamento de REEE domésticos na Malásia, visando contribuir para o avanço das cidades inteligentes sustentáveis. Nesse sentido, os autores desenvolveram um sistema automatizado a partir de um coletor de resíduos com sensores que notifica a coleta e posteriormente auxilia os usuários finais públicos no processo de descarte dos resíduos.

Nas pesquisas de Anand et al. (2018), Misra et al. (2018), Wen et al. (2018) e Jacob et al. (2019) foram desenvolvidos sistemas inteligentes de gerenciamento de resíduos urbanos que utilizam caixas de coletas com sensores para indicar a condição e notificar a necessidade de coleta, além de orientar as melhores rotas e reduzir as viagens entre a coleta e a destinação final dos resíduos. Ambos os estudos visam maximizar a recuperação de resíduos que possuem valor de mercado e potencial de reciclagem e são descartados nas lixeiras coletoras misturados com os demais resíduos.

Buscando auxiliar no processo de realocação de caminhões de coletas de caixas inteligentes de resíduos sólidos ao longo do tempo, Shah et al. (2018) estudaram o uso de análise estocásticas. Também nessa linha, Idwan et al. (2019) elaboraram um algoritmo baseado em outro criado anteriormente pelo grupo que visa auxiliar no traçado ótimo das rotas de coleta de resíduos, facilitando o processo de gerenciamento dos resíduos sólidos urbanos, em região metropolitana.

Chakravarthi et al. (2019) descrevem quatro formas de gerenciamento de resíduos sólidos para cidade de Amaravati, na Índia, que apresenta crescimento populacional elevado, a partir de um questionário aplicado às partes envolvidas e de consulta bibliográficas. Os autores concluíram que o sistema pneumático é o mais adequado para a realidade da área estudada, apontando para uma busca por sistemas altamente tecnológicos e sustentáveis.

Por fim, Xue et al. (2019) abordaram uma análise comparativa entre a coleta inteligente usando IoT e a coleta informal de resíduos sólidos urbanos na China, avaliando aspectos econômicos e sociais inerentes aos dois modelos. Os autores 
relatam vantagens da coleta inteligente em relação a coleta informal, de modo que essas vantagens tornam a coleta inteligente com o potencial de integrar ou substituir a coleta informal.

\section{Conclusão}

A revisão sistemática com análise bibliométrica mostrou-se um método eficaz para a compreensão do panorama técnico-científico da relação e da aplicação mundial da IoT na gestão de resíduos sólidos, bem como para nortear futuras pesquisas. Isto ficou comprovado pelo escasso número de publicações de artigos existentes na base de dados adotada e no recorte temporal analisado. No entanto, constatou-se que os estudos científicos vêm aumentando, ao longo dos anos, principalmente a partir de 2018, evidenciando que a área de estudo se encontra em ascensão.

Verificou-se que o tema "IoT na gestão de resíduos sólidos" tem sido estudado com foco na utilização de tecnologias de IoT para automatização de lixeiras, a fim de melhorar o gerenciamento. Essa automação de caixa coletora de resíduos, que notifica o momento de realizar a coleta dos resíduos para serem conduzidos até a destinação final, possibilita a otimização das rotas e auxilia no transporte dos resíduos da coleta até a destinação final, consequentemente aumentando a eficiência da utilização de recursos naturais e financeiros.

Tendo em vista esses aspectos, é de grande relevância o desenvolvimento de pesquisas que busquem novas descobertas e analisem o emprego das tecnologias da IoT para auxiliar no gerenciamento de resíduos, construindo cidades inteligentes e sustentáveis.

\section{Referências}

Agência USP de Gestão da Informação Acadêmica. 2016. Disponível em: https://www.aguia.usp.br/apoio-

pesquisador/indicadores-pesquisa/lista-

indicadores-bibliometricos/. Acesso em: 27 jun. 2020.

Alqahtani, F.; Al-Makhadmeh, Z.; Tolba, A.; Said, W. 2020. Internet of things-based urban waste management system for smart cities using a Cuckoo search algorithm. Cluster Computing, 23, 1769-1780. DOI: 10.1007/s10586-020-03126-x

Anagnostopoulos, T.; Zaslavsky, A.; Sosunova, I.; Fedchenkov, P.; Medvedev, A.; Ntalianis, K.; Skourlas, C.; Rybin, A.; Khoruznikov, S. 2018. A stochastic multi-agent system for Internet of Things-enabled waste management in smart cities. Waste
Management \& Research, 1-9. DOI: 10.1177/0734242x18783843

Anand, A.; Verma, S.; Padmini, T. N. 2018. Realtime garbage monitoring system with periodic updation of database server and notification using IOT and GSM. Journal of Advanced Research in Dynamical and Control Systems, 10, 3, 1036-1039.

Atayero, A. A.; Williams, R.; Badejo, J. A.; Popoola, S. I. 2019. Cloud Based IoT-enabled solid waste monitoring system for smart and connected communities. International Journal of Civil Engineering and Technology, 10, 2, 2308-2315.

Batagan, L. 2011. Smart Cities and Sustainability Models. Informatica Economica, 15, 3, 8087.

Bhanot, N.; Sharma, V. K.; Parihar, A. S.; Sharma, M.; Yadav, M.; Gupta, H. 2019. A conceptual framework of internet of things for efficient municipal solid waste management and waste to energy implementation. International Journal of Environment and Waste Management, 23, 4, 410-432. DOI: 10.1504/IJEWM.2019.099997

Bong, C. P. C.; Lim, L. Y.; Lee, C. T.; Fan, Y. V.; Klemešb, J. J. 2018. The role of smart waste management in smart agriculture. Chemical Engineering Transactions, 70, 937-942. DOI: 10.3303/CET1870157

Chakravarthi, G. K.; Chandra, D. S.; Asadi, SS. 2019. Smart solid waste management in new Capital City Amaravathi. International Journal of Recent Technology and Engineering, 7, 6C2, 882-887.

Couth, R.; Trois, C. 2012. Sustainable waste management in Africa through CDM projects. Waste Management, 32, 11, 21152125. DOI: 10.1016/j.wasman.2012.02.022

Dhana Shree, K.; Janani, B.; Reenadevi, R.; Rajesh, R. 2019. Garbage monitoring system using smart bins. International Journal of Scientific \& Technology Research, 8, 11, 1921-1925.

Diniz, C. C. 2019. Corrida científica e tecnológica e reestruturação produtiva: impactos geoeconômicos e geopolíticos. Revista Brasileira de Estudos Urbanos e Regionais, $21, \quad 2$, 241-257. DOI: 10.22296/2317$1529.2019 \mathrm{v} 21 \mathrm{n} 2 \mathrm{p} 241$

Ferrari, F.; Striani, R.; Minosi, S.; Fazio, R.; Visconti, P.; Patrono, L.; Catarinucci, L.; Corcione, C. E.; Greco, A. 2020. An innovative IoT-oriented prototype platform for the management and valorisation of the organic fraction of municipal solid waste. 
Journal of Cleaner Production, 247, 119618. DOI: $10.1016 /$ j.jclepro.2019.119618

Gaddam, M.; Thatha, V. D.; Kavuluri, S. R.; Popuri, G. K. 2018. Smart garbage collection management system. International Journal of Engineering \& Technology, 7, 2.7, 193-196. DOI: 10.14419/ijet.v7i2.7.10291

Galvão, T. F.; Pereira, M. G. 2014. Revisões sistemáticas da literatura: passos para sua elaboração. Epidemiol. Serv. Saude, 23, 1, 183-184. DOI: $\quad 10.5123 / \mathrm{S} 1679-$ 49742014000100018

Garrido-Hidalgo, C.; Ramirez, F. J.; Olivares, T.; Roda-Sanchez, L. 2020. The adoption of Internet of Things in a Circular Supply Chain framework for the recovery of WEEE: The case of Lithium-ion electric vehicle battery packs. Waste Management, 103, 32-44. DOI: 10.1016/j.wasman.2019.09.045

Gašová, M.; Gašo, M.; Štefánik, A. 2017. Advanced industrial tools of ergonomics based on Industry 4.0 concept. Procedia Engineering, 192, 219-224. DOI: 10.1016/j.proeng.2017.06.038

Gu, F.; Ma, B.; Guo, J.; Summers, P. A.; Hall, P. 2017. Internet of things and Big Data as potential solutions to the problems in waste electrical and electronic equipment management: an exploratory study. Waste Management, 68, 434-448. DOI: 10.1016/j.wasman.2017.07.037

Idwan, S.; Mahmood, I.; Zubairi, J. A.; Matar, I. 2019. Optimal management of solid waste in smart cities using internet of things. Wireless Personal Communications, 110, 485-501. DOI: $10.1007 / \mathrm{s} 11277-019-06738-8$

Ismail, N. A.; Majid, N. A. A.; Hassan, S. A. 2019. IoT-based smart solid waste management system a systematic literature review. International Journal of Innovative Technology and Exploring Engineering, 8, 8, 1456-1462.

Jacob, P. M.; Kabeer, R.; Nair, N.; Saji, S. V.; Meenakshi; Varghese, R. R. 2019. An intelligent solid waste management system using internet of Things (IoT). International Journal of Recent Technology and Engineering, 8, 2S4, 120-125. DOI: 10.35940/ijrte.B1021.0782S419

Kang, K. D.; Kang, H.; Ilankoon, I. M. S. K.; Chong, C. Y. 2020. Electronic waste collection systems using Internet of Things (IoT): household electronic waste management in Malaysia. Journal of Cleaner Production, 119801 . DOI: 10.1016/j.jclepro.2019.119801
Katz, J. S.; Martin, B. R. 1997. What is research collaboration? Research Policy, 26, 1, 1-18. DOI: $10.1016 / \mathrm{S} 0048-7333(96) 00917-1$

Lacerda, F.; Lima-Marques, M. 2015. Da necessidade de princípios de arquitetura da informação para a Internet das Coisas. Perspectivas em Ciência da Informação, 20, 2, 158-171. DOI: 10.1590/1981-5344/2356

Liu, W.; Wang, J.; Li, C.; Chen, B.; Sun, Y. 2019. Using Bibliometric Analysis to Understand the Recent Progress in Agroecosystem Services Research. Ecological Economics, 156, 293-305. DOI: 10.1016/j.ecolecon.2018.09.001

Logan, M.; Safi, M.; Lens, P.; Visvanathan, C. 2019. Investigating the performance of internet of things based anaerobic digestion of food waste. Process Safety and Environmental Protection, 127, 277-287. DOI: 10.1016/j.psep.2019.05.025

Lu, J.-W.; Chang, N.-B.; Liao, L. 2013. Environmental informatics for solid and hazardous waste management: advances, challenges, and perspectives. Critical Reviews in Environmental Science and Technology, 43, 15, 1557-1656. DOI: 10.1080/10643389.2012.671097

Machado Junior, C.; Souza, M. T. S.; Parisotto, I. R. S.; Palmisano, A. 2016. As leis da bibliometria em diferentes bases de dados científicos. Revista de Ciências e Administração, 18, 44, 111-123. DOI: 10.5007/2175-8077.2016v18n44p111

Misra, D.; Das, G.; Chakrabortty, T.; Das, D. 2018. An IoT-based waste management system monitored by cloud. Journal of Material Cycles and Waste Management, 20, 3, 15741582. DOI: $10.1007 / \mathrm{s} 10163-018-0720-y$

Moher, D.; Liberati, A.; Tetzlaff, J.; Altman, D. G. 2010. Preferred reporting items for systematic reviews and meta-analyses: The PRISMA statement. International Journal of Surgery, 8, 5, 336-341. DOI: 10.1016/j.ijsu.2010.02.007

Monishan, M. V.; Pankajavalli, P. B.; Karthick, G. S. 2019. Implementation of novel optimal scheduling and routing algorithm on IoTbased garbage disposal system. International Journal of Innovative Technology and Exploring Engineering, 8, 7, 768-772.

Najmi, A.; Rashidi, T. H.; Abbasi, A.; Travis Waller, S. 2017. Reviewing the transport domain: an evolutionary bibliometrics and network analysis. Scientometrics, 110, 2, 843-865. DOI: 10.1007/s11192-016-2171-3

Newman, M. E. J. 2004. Coauthorship networks and patterns of scientific collaboration. Proceedings of the National Academy of 
Sciences, 101 (Suppl. 1), 5200-5205. DOI: 10.1073/pnas.0307545100

Nizetic, S.; Solic, P.; López-de-Ipiña, D.; Patrono, L. 2020. Internet of Things (IoT): Opportunities, issues and challenges towards a smart and sustainable future. Journal of Cleaner Production, 274, 20, 122877. DOI: 10.1016/j.jclepro.2020.122877

Pantaleão, P. F.; Veiga, H. M. S. 2019. Bem-estar no trabalho: revisão sistemática da literatura nacional na última década. HOLOS, 35, 5, 124. DOI: $10.15628 /$ holos. 2019.7570

Pardini, K.; Rodrigues, J. J. P. C.; Kozlov, S.; Kumar, N.; Furtado, V. 2019. IoT-based solid waste management solutions: a survey. Journal of Sensor and Actuator Networks, 8, 5. DOI: $10.3390 /$ jsan 8010005

Pluye, P.; Hong, Q. N. 2014. Combining the Power of Stories and the Power of Numbers: Mixed Methods Research and Mixed Studies Reviews. Annual Review of Public Health, 35 , 1, 29-45. DOI: 10.1146/annurevpublhealth-032013-182440

Rodelas, N. C.; Perez, V. O.; Salvador Jr., J. E.; Lazaro, J. P.; Rubio, A. J. M.; Cabreros, A. D.; Cruz, E. B. M. D. 2020. Solid waste management and collection system in metro manila with dijkstra algorithm and internet of things. International Journal of Scientific \& Technology Research, 9, 4, 2063-2067.

Saraiva, P. P.; Camara, I. P.; Ribeiro, L. A.; Silva, T. L. 2019. O uso de tecnologias como estratégias na construção de cidades mais inteligentes e sustentáveis. Gestão \& Regionalidade, 35, 105, 184-199. DOI: 10.13037/gr.vol35n105.5292

Shah, P. J.; Anagnostopoulos, T.; Zaslavsky, A.; Behdad, S. 2018. A stochastic optimization framework for planning of waste collection and value recovery operations in smart and sustainable cities. Waste Management, 78, 104-114.

DOI: 10.1016/j.wasman.2018.05.019

Sharma, M.; Joshi, S.; Kannan, D.; Govindan, K.; Singh, R.; Purohit, H. C. 2020. Internet of Things (IoT) adoption barriers of smart cities' waste management: An Indian context. Journal of Cleaner Production, 270, 10, 122047. DOI: 10.1016/j.jclepro.2020.122047

Simão, A. D. S.; Alcoforado, L. F.; Longo, O. C.; Santos, D. A., Santos, F., Silva, A. D.; Junior Meirelles, J. C. 2019. Impactos da indústria 4.0 na construção civil brasileira. Brazilian Journal of Development, 5, 19670-19685. DOI: $10.34117 /$ bjdv5n10-210
Srikanth, C. S.; Rayudu, T. B.; Radhika, J.; Anitha, R. 2019. Smart waste management using internet-of-things (IoT). International Journal of Innovative Technology and Exploring Engineering, 8, 9, 2518-2522. DOI: 10.35940/ijitee.G5334.078919

Vaisali, G.; Bhargavi, K. S.; Kumar, S. 2017. Smart solid waste management system by IoT. International Journal of Mechanical Engineering and Technology, 8, 12, 841-846.

Van Eck, N. J.; Waltman, L. 2010. Software survey: VOSviewer, a computer program for bibliometric mapping. Scientometrics, 84, 2 , 523-538. DOI: 10.1007/s11192-009-0146-3.

Van Eck, N. J.; Waltman, L. 2017. Citation-based clustering of publications using CitNetExplorer and VOSviewer. Scientometrics, 111, 1053-1070. DOI: 10.1007/s11192-017-2300-7.

Velvizhi, G.; Shanthakumar, S.; Das, B.; Pugazhendhi, A.; Priya, T. S.; Ashok, B.; Nanthagopal, K.; Vignesh, R.; Karthick, C. 2020. Biodegradable and non-biodegradable fraction of municipal solid waste for multifaceted applications through a closed loop integrated refinery platform: Paving a path towards circular economy. Science of the Total Environment, 731, 138049. DOI: 10.1016/j.scitotenv.2020.138049

Wen, Z.; Hu, S.; Clercq, D.; Beck, M. B.; Zhang, H.; Zhang, H.; Fei, F.; Liu, J. 2018. Design, implementation, and evaluation of an Internet of Things (IoT) network system for restaurant food waste management. Waste Management, 73, 26-38. DOI: 10.1016/j.wasman.2017.11.054

Xue, Y.; Wen, Z.; Bressers, H.; Ai, N. 2019. Can intelligent collection integrate informal sector for urban resource recycling in China? Journal of Cleaner Production, 208, 307-315. DOI: 10.1016/j.jclepro.2018.10.155

Zago, V. C. P.; Barros, R. T. V. 2019. Gestão dos resíduos sólidos orgânicos urbanos no Brasil: do ordenamento jurídico à realidade. Engenharia Sanitária e Ambiental, 24, 2, 219228. DOI: $10.1590 / \mathrm{S} 1413-41522019181376$

Zhou, G.; Gu, Y.; Wu, Y.; Gong, Y.; Mu, X.; Han, H.; Chang, T. 2020. A systematic review of the deposit-refund system for beverage packaging: operating mode, key parameter and development trend. Journal of Cleaner Production, 251, $119660 . \quad$ DOI: 10.1016/j.jclepro.2019.119660 